TRANSACTIONS OF THE

AMERICAN MATHEMATICAL SOCIETY

Volume 354, Number 11, Pages 4493-4504

S 0002-9947(02)03102-1

Article electronically published on July 2, 2002

\title{
CLASSIFICATION OF COMPACT COMPLEX HOMOGENEOUS SPACES WITH INVARIANT VOLUMES
}

\author{
DANIEL GUAN
}

\begin{abstract}
We solve the problem of the classification of compact complex homogeneous spaces with invariant volumes (see Matsushima, 1961).
\end{abstract}

\section{INTRODUCTION}

We call a $2 n$-dimensional manifold $M$ a complex homogeneous space with invariant volume if there is a complex structure and a nonzero $2 n$-form on $M$ such that a transitive Lie transformation group keeps both the complex structure and the $2 n$-form invariant. There are many papers published in the direction of classification of such manifolds, e.g., [4], 6], [7, [8, [9], 11, [12, [16, 19], 20, 27, 29], [30, 35] and the references there (see also [2] [5], 10], [17, [11, 12], 14, 33], 34 for related topics involving compact complex homogeneous spaces). In this paper, we shall deal with the compact case and finish the classification up to certain better-understood building blocks.

In this paper, we shall always deal with compact manifolds except those manifolds in the Preliminaries and in Theorem 1.

After [30, not much has been done for the classification of compact complex homogeneous spaces with invariant volumes until very recently. Two breakthroughs in [6] are: first, the proof that the Hano-Kobayashi fiberation (we might also call it the Ricci form reduction) is holomorphic and is the same as the anticanonical fibering in the compact case; second, the classification of compact complex homogeneous spaces with invariant pseudo-Kähler structures (see [6], 19] and [11], [12], also [13]).

In [19] Huckleberry observed that one can handle the pseudo-Kähler case by using methods from symplectic geometry.

Huckleberry's method was used in [11], [12] to obtain following theorem:

Proposition 1. Every compact homogeneous complex manifold with a 2-cohomology class $\omega$ such that $\omega^{n}$ is not zero in the top cohomology is a product of a rational homogeneous space and a complex parallelizable solv-manifold with a symplectic structure which is right-invariant in its universal covering.

Received by the editors September 28, 2001 and, in revised form, April 21, 2002.

2000 Mathematics Subject Classification. Primary 53C30, 32M10, 32M05 14M17; Secondary 14M20, 53C10, 53C56.

Key words and phrases. Invariant volume, homogeneous, product, fiber bundles, complex manifolds, parallelizible manifolds, discrete subgroups, classifications.

Supported by NSF Grants DMS-9401755 and DMS-9627434.

(C)2002 American Mathematical Society 
This generalized the result of [5] for the Kähler case (one does not assume that the Kähler form is invariant).

For a general compact complex homogeneous space with an invariant volume, the symplectic method does not apply. However, our original method (see [20], [30] and [6]) gives a classification (see Theorem 4).

Main Theorem 1. Every compact complex homogeneous space with an invariant volume form is a principal homogeneous complex torus bundle over the product of a projective rational homogeneous space and a parallelizable manifold. Conversely, every compact complex homogeneous space that is a complex homogeneous torus bundle over a product of a projective rational homogeneous space and a complex parallelizable manifold admits a transitive real Lie group $G$, acting on $M$ by holomorphic transforms and preserving a volume form on $M$.

It is this theorem that motivates the torus bundle type of structure theorems in [14.

For more details of the Main Theorem 1, one might look at sections 3, 4 and 5 . We actually give a very explicit construction of this kind of manifold as a complex quotient manifold of a product of a manifold in 35] and a manifold in 34] by the anti-diagonal action of a complex torus which acts on both manifolds and is in the center of the latter manifold. See section 5 for the details.

The proof of Theorem 3 is critical. By applying a straightforward argument in the proof of Theorem 3, we can obtain the second part of the Main Theorem 3 in 15.

Theorem 1 may be applied to the noncompact case and Theorem 5 is stronger than our Main Theorem 1 in some sense. For some technical reasons, we do not state them here but refer the interested readers to the detailed sections.

We also note that every compact complex homogeneous space $M$ with a 2cohomology class such that its top power is nonzero in the top cohomology group, admits a transitive real Lie transformation group $G$, which acts on $M$ by holomorphic transforms and preserves a volume form. It is also well-known that all simply connected compact complex homogeneous spaces studied in 35] have invariant volumes since the transitive group can be chosen to be a compact Lie group.

In [30] Matsushima considered the special case of a semisimple group action. He proved:

Proposition 2. If $G / H$ is a compact complex homogeneous space with a $G$-invariant volume and $G$ semisimple, then $G / H$ is a holomorphic fiber bundle over a rational homogeneous space with a complex reductive parallelizable manifold as a fiber.

Applying our Main Theorem 1 to this situation, we immediately obtain that the result of Matsushima can be generalized to the case when $G$ is reductive. Moreover, we have the following stronger result.

Main Theorem 2. Assume that $G / H$ is a compact complex homogeneous space with a $G$-invariant volume and that $G$ is reductive. Then $G / H$ is a principal holomorphic torus bundle over the product of a projective rational homogeneous space and a complex parallelizable homogeneous space of a semisimple complex Lie group.

Theoretically our arguments can be applied to solve a more general problem of classifying all compact complex homogeneous spaces up to some better-understood building blocks. We shall leave it to [14]. 


\section{Preliminaries}

2.1. Special Compact Complex Homogeneous Spaces. A rational homogeneous manifold $Q$ is a compact complex manifold that can be realized as a closed orbit of a linear algebraic group in some projective space. Equivalently, $Q=S / P$ where $S$ is a complex semisimple Lie group and $P$ is a parabolic subgroup, i.e., a subgroup of $S$ that contains a maximal connected solvable subgroup (Borel subgroup). Every homogeneous rational manifold is simply-connected and is therefore an orbit of a compact group. In general, a quotient $K / L$ with $K$ compact and semisimple carries a $K$-invariant complex structure that is projective algebraic if and only if $L$ is the centralizer $C(T)$ of a torus $T \subset K$.

A parallelizable complex manifold is a compact quotient of a complex Lie group by a discrete subgroup. It is a solv-manifold or nil-manifold according as the complex Lie group is solvable or nilpotent. In the same way, we can define reductive parallelizable manifolds and semisimple parallelizable manifolds.

2.2. Generalized Tits Fibrations. In this subsection we recall some basic results on a generalization of the Tits fibration, introduced by A. Huckleberry and E. Oeljeklaus [21. It coincides with a fibration considered by Hano [16] in case the isotropy group is connected. We call it the $\mathcal{G}$-anticanonical fibering as in 21] or the $H O T$-fibration as in [6]. Let $M=G / H, H^{0}$ be the identity component of $H$ and $\operatorname{Norm}_{G}\left(H^{0}\right)$ the normalizer of $H^{0}$ in $G$. Then we have:

Proposition 3. Let $G$ be a connected real Lie group acting almost effectively and transitively as a group of holomorphic transformations on the complex manifold $M=G / H$ and let $G / H \rightarrow G / J$ be the HOT-fibration.

Then

1. $J=\left\{k \in \operatorname{Norm}_{G}\left(H^{0}\right) ; R(k): G / H^{0} \rightarrow G / H^{0}, g H^{0} \rightarrow g k H^{0}\right.$, holomorphic $\}$ where $G / H^{0}$ carries the complex structure induced by $G / H^{0} \rightarrow G / H$. In particular, we have $J \subset \operatorname{Norm}_{G}\left(H^{0}\right)$.

2. $J / H^{0}$ is a complex Lie group and $G / H^{0} \rightarrow G / J$ is a holomorphic $J / H^{0}$ principal fiber bundle. In particular, the fibering $G / H \rightarrow G / J$ is locally holomorphically trivial.

3. If $G$ is a connected complex Lie group and $H$ a closed complex subgroup, then $J=\operatorname{Norm}_{G}\left(H^{0}\right)$. Thus for a complex Lie group $G$, the HOT-fibration coincides with the Tits fibration.

If $G$ is a complex Lie group and $H \subset G$ is a closed complex subgroup, then we have the normalizer fibration $G / H \rightarrow G / N$, where $N=N_{G}\left(H^{0}\right)$. Let $\mathcal{G}$ and $\mathcal{H}$ denote the Lie algebras of $G$ and $H$, respectively. The base space $G / N$ is realized as the $\operatorname{Ad}(G)$-orbit of the subspace $\mathcal{H}$ in the Grassmann manifold of subspaces of $\mathcal{G}$ that have the same dimension as that of $\mathcal{H}$. If $G / H$ is compact, then $G / N$ is a rational homogeneous manifold and $N / H$ is a compact parallelizable homogeneous manifold.

2.3. More on HOT Fibrations. We also recall Tits' result on the fibration of compact homogeneous spaces:

Proposition 4. Let $G$ be a connected complex Lie group and $H$ a closed complex subgroup such that $G / H$ is compact. Then $G / \operatorname{Norm}_{G}\left(H^{0}\right)$ is a rational homogeneous space and $\operatorname{Norm}_{G}\left(H^{0}\right) / H$ is connected and parallelizable. Moreover, if $G / H \rightarrow G / R$ is a holomorphic fibration with parallelizable fiber $R / H$, then 
$R \subset \operatorname{Norm}_{G}\left(H^{0}\right)$; if, in addition, the base $G / R$ is rational homogeneous, then $R=\operatorname{Norm}_{G}\left(H^{0}\right)$.

Moreover, if $G$ is a real Lie group such that $G / H$ is a compact complex manifold with $G$ acting holomorphically and almost effectively, then by complexifying the vector fields corresponding to the Lie algebra, we can see that there exists a connected complex Lie group $G^{\mathbf{C}}$ such that $G \subset G^{\mathbf{C}}$ and $G / H=G^{\mathbf{C}} / H^{\mathbf{C}}$.

In general, $G^{\mathbf{C}}$ might not be a complexification of $G$, but we can always choose $G^{\mathbf{C}}$ such that $\mathcal{G}^{\mathbf{C}}=\mathcal{G}+i \mathcal{G}$.

From the definition of the HOT-fibration [21, 1.7], it is easy to observe that $G / H$ and $G^{\mathbf{C}} / H^{\mathbf{C}}$ have the same HOT-fibration:

Proposition 5. Let $G$ be a connected real Lie group acting almost effectively and transitively as a group of holomorphic transformations on the compact, complex manifold $G / H=G^{\mathbf{C}} / H^{\mathbf{C}}$.

Let $G / H \rightarrow G / J$ denote the HOT-fibration of $G / H$. Then the action of $G^{\mathbf{C}}$ on $G / H$ preserves this fibration. Moreover, let $G^{\mathbf{C}} / H^{\mathbf{C}} \rightarrow G^{\mathbf{C}} / J^{\mathbf{C}}$ denote the Tits fibration. Then $J=J^{\mathbf{C}} \cap G$, i.e., $G / J=G^{\mathbf{C}} / J^{\mathbf{C}}$. Thus, for compact $G / H$, the HOT-fibration and the Tits fibration are the same, and the HOT-fibration does not depend on the choice of $G$. In particular, $J$ is connected and $G / J$ is rational homogeneous.

2.4. Hano-Kobayashi Fibration. Next we want to discuss the Hano-Kobayashi fibration. We shall call it the HK-fibration (or Ricci form reduction, or the canonical fibration). Let $M$ be a complex manifold and $\omega=K(z, \bar{z}) d z^{1} \wedge \cdots \wedge d z^{n} \wedge d \bar{z}^{1} \wedge$ $\cdots \wedge d \bar{z}^{n}$ be an invariant volume form. We also set

$$
R_{i \bar{j}}=\frac{\partial^{2} \log K}{\partial z^{i} \partial \bar{z}^{j}}
$$

and

$$
\chi=i \sum R_{i \bar{j}} d z^{i} \wedge d \bar{z}^{j}
$$

Then $\chi$ is called the Ricci form of $M$. We recall the main result on the HK-fibration for homogeneous complex manifolds (see [20]):

Proposition 6. Let $M$ be a connected complex manifold and $G$ a connected real Lie group acting holomorphically on $M$. Assume, moreover, that $M=G / H$ admits a G-invariant volume element $\omega$ and denote by $\chi$ the associated Ricci form of $M$.

Then there exists a unique closed subgroup $I$ of $G$ containing $H$ and a nondegenerate closed two-form $\hat{\chi}$ on $G / I$ such that

1. $G / I$ is a homogeneous symplectic manifold with respect to $\hat{\chi}$.

2. The fiber $I / H$ of the projection $G / H \rightarrow G / I$ is a complex connected submanifold of $G / H$ and $\left.\chi\right|_{I / H}=0$.

3. The pull-back of $\hat{\chi}$ to $M$ is equal to $\chi$.

4. If $I / H$ is compact, then it is (complex) parallelizable.

In [11, 12] we notice that the definition of the HOT-fibration is global $(\mathrm{J} / \mathrm{H}$ might not be connected, for example) and the definition of the HK-fibration is local (see (2) in Proposition 6). Therefore, we might call the fibration in Proposition 5 the global HOT-fibration (or global anticanonical fibration) and define the local $H O T$-fibration by the fibering $G / H \rightarrow G / J_{1}$ where $J_{1}$ is the minimal closed and open subgroup of $J$ that contains $H$ (hence $J_{1} / H$ is connected). We might also call 
the fibration in Proposition 6 the local HK-fibration (or local canonical fibration) and define the global $H K$-fibration by the fibering $G / H \rightarrow G / I_{1}$ where $I_{1}=\{g \in$ $G \mid g^{*} \operatorname{div}(j X)=\operatorname{div}(j X)$ for all $\left.X \in \mathcal{G}\right\}$, where $j$ is the complex structure and div is the divergence (see [20, p. 236] for the proof of Theorem A).

2.5. Koszul Algebra. In the rest of this paper, we shall frequently use arguments at the Lie algebra level.

First we recall the following result due to Koszul [27]:

Proposition 7. Let $G$ be a real Lie group and $H$ a closed subgroup. Then $G / H$ admits a $G$-invariant complex structure if and only if there exists an endomorphism $j$ of $\mathcal{G}$ such that for all $x, y \in \mathcal{G}, r \in H$ we have:

$$
\begin{aligned}
& j \mathcal{H} \subset \mathcal{H}, \\
& j^{2} x=-x(\bmod \mathcal{H}), \\
& \operatorname{Ad} r(j x)=j \operatorname{Ad} r(x)(\bmod \mathcal{H}), \\
& {[j x, j y]=j[j x, y]+j[x, j y]+[x, y](\bmod \mathcal{H}) .}
\end{aligned}
$$

Notice that $j$ is only determined modulo $\mathcal{H}$; so we might assume that $j \mathcal{H}=0$. If we assume that $M=G / H$ has a $G$-invariant volume form $\omega$, we let

$$
\phi(x)=\operatorname{tr}_{\mathcal{G} / \mathcal{H}}(\operatorname{ad} j x-j \circ \operatorname{ad} x), \quad x \in \mathcal{G} .
$$

Then $\phi(x)=\operatorname{div}(j X)$ where $X$ is the holomorphic vector field corresponding to $x$ (see [20, p. 236]). From [27] (see also [20, Lemma 3.2]):

Proposition 8. The Ricci form associated with $\omega$ is given by the formula

$$
\chi(x, y)=\phi([x, y]), \quad x, y \in \mathcal{G} .
$$

Moreover, the Ricci form satisfies for $x, y, z \in \mathcal{G}$,

$$
\begin{aligned}
& \chi(j x, j y)=\chi(x, y), \\
& \chi([x, y], z)+\chi([y, z], x)+\chi([z, x], y)=0, \\
& \chi(\mathcal{G}, \mathcal{H})=0 .
\end{aligned}
$$

With the notation of this subsection, we have an expression of global HOTfibration $G / H \rightarrow G / J$ with

$$
J=\left\{g \in G \mid g H^{0} g^{-1}=H^{0}, j \circ \operatorname{Ad}(g)=\operatorname{Ad}(g) j \circ(\bmod \mathcal{H})\right\} .
$$

Actually, this fibration is the same as the fibration given by the anticanonical line bundle in the compact case and an expression of the global HK-fibration $G / H \rightarrow$ $G / I_{1}$ with

$$
\begin{aligned}
& I_{1}=\left\{g \in G \mid \operatorname{tr}_{\mathcal{G} / \mathcal{H}}(\operatorname{ad}(j(\operatorname{Ad} g(x)-x))-j \circ \operatorname{ad}(\operatorname{Ad} g(x)-x))=0\right. \\
& \quad \text { for all } x \in \mathcal{G}\}
\end{aligned}
$$

This comes from the proof of Theorem A in [20, p. 236] (see also Lemmas 3.1 and 3.2 there). The condition in the bracket is the same as $\phi(x)=\phi(\operatorname{Ad} x)$. We observe that $x$ and $y$ in $G / H$ define the same point in $G / I_{1}$ if and only if $f_{X}(x)=f_{X}(y)$ (that is, if $y=g x$, then $\operatorname{div} j X=\operatorname{div} j \operatorname{Ad} g(X)$ ) for all $X \in \mathcal{G}$ regarding as right invariant vector fields on $G$, where $f_{X}$ is $\operatorname{div} j X$, the divergence of the vector field $j X$. In this situation, $\operatorname{div} j[X, Y]=\operatorname{div} j \operatorname{Ad} g([X, Y])=\operatorname{div} j[\operatorname{Ad} g(X), \operatorname{Ad} g(Y)]$, i.e., the Ricci form comes from a 2 -form on $G / I_{1}$. 
Also, for any $x, y \in \mathcal{G}$, we have $\chi(x, y)=\operatorname{div}\left(j\left(\mathrm{~L}_{X}(Y)\right)\right)$ where $X, Y$ are the holomorphic vector fields corresponding to $x, y$.

From [16] we know that the Lie algebra $\mathcal{J}$ of $J$ can also be described as follows: Let $\mathcal{G}_{-}=\left\{x+\left.i j x\right|_{x \in \mathcal{G}}\right\}$. Then $\mathcal{H}=\mathcal{G} \cap \mathcal{G}_{-}$(this follows from the fact that $j x$ is the same as $j x$ in the holomorphic tangent space at the considered point and hence $x+i j x$ is $x+i i x=x-x=0)$ and $\mathcal{J}=\mathcal{G} \cap \operatorname{norm}_{\mathcal{G}} \mathbf{C}\left(\mathcal{G}_{-}\right)$.

2.6. Representation Theory. Here we collect some results that we need from the representation theory of the semisimple Lie algebras (Cf. [22, pp. 67-69, 113]). Let $s$ be a semisimple Lie algebra, $t$ a Cartan subalgebra, $\Delta$ an ordered root system, $\Delta^{+}$the positive roots. We let $\delta=\frac{1}{2} \sum_{\alpha \in \Delta^{+}} \alpha$ and $\left\{\alpha_{1}, \cdots, \alpha_{l}\right\}$ be the set of simple roots. We also let $\left\{H_{1}, \cdots, H_{l}\right\} \subset t$ be a set of elements dual to the simple roots such that $\frac{2\left(H_{i}, \alpha_{j}\right)}{\left(\alpha_{j}, \alpha_{j}\right)}=\delta_{i j}$. We have:

Proposition 9. Let s be a semisimple Lie algebra. Then:

1. An element in $t$ is a highest weight for an irreducible representation if and only if it can be expressed as $\sum a_{i} H_{i}$ with $a_{i}$ nonnegative integers.

2. $\delta=\sum H_{i}$.

3. $H_{i}=\sum_{j} a_{i j} \alpha_{i}$ with positive $a_{i j}$.

4. Let $\pi_{i}$ be the representation corresponding to $H_{i}$. Then the unique irreducible representation with highest weight as in (1) is a submodule of $\otimes\left(\pi_{i}\right)^{a_{i}}$ generated by the highest weight vector which is the tensor product of the highest weight vectors of $\pi_{i}$.

The statements (1), (2) and (4) come from the standard representation theory, while (3) can be found in [22, p. 69].

\section{Four Fibrations}

In this section, we shall prove the following generalization of a theorem in [6]:

Theorem 1. For any complex homogeneous space $G / H$ with an invariant volume, compact or noncompact, $J \subset I_{1}$.

Proof. Let $g \in J$ and $x \in \mathcal{G}$. Then $j \operatorname{Ad} g(x)=\operatorname{Ad} g(j x)+h$ for some $h \in \mathcal{H}$. Therefore,

$$
\begin{aligned}
\operatorname{ad}(j & (\operatorname{Ad} g(x)-x))-j \circ \operatorname{ad}(\operatorname{Ad} g(x)-x) \\
& =\operatorname{ad}(\operatorname{Ad} g(j x)-j x)+\operatorname{ad} h-j \circ((\operatorname{Ad} g-1)(\operatorname{ad} x)) \\
& =((\operatorname{Ad} g-1)(\operatorname{ad} j x))+\operatorname{ad} h-((\operatorname{Ad} g-1)(j \operatorname{ad} x))+([\operatorname{Ad} g, j](\operatorname{ad} x)) \\
& =((\operatorname{Ad} g-1)(\operatorname{ad} j x-j \operatorname{ad} x))+\operatorname{ad} h+([\operatorname{Ad} g, j](\operatorname{ad} x)) .
\end{aligned}
$$

Here we also regard $\operatorname{Ad} g$ as a map acting on the matrices ad $y$ and $j \operatorname{ad} x$, i.e., $\operatorname{Ad} g(A)=m(g) A(m(g))^{-1}$ for any matrix $A$ with $m(g)$ being the transformation matrix of $\operatorname{Ad} g$ on $\mathcal{G}$ with respect to a given basis of $\mathcal{G}$. We notice that $\operatorname{ad}(j x)-j \operatorname{ad} x$ and $\operatorname{Ad} g$ leave $\mathcal{H}$ invariant. Therefore, by $\operatorname{tr}(\operatorname{Ad} g-1)(A)=\operatorname{tr}\left(m(g) A(m(g))^{-1}-\right.$ $A)=0$ for any matrix $A$, the trace of the first term vanishes. Since $M$ admits an invariant volume form, we have that the trace of the second term vanishes as well. Now we notice that by the definition of the HOT-fibration $[\operatorname{Ad} g, j] \mathcal{G} \subset \mathcal{H}$, we have

$$
([\operatorname{Ad} g, j] A) y=[m(g), j] A(m(g))^{-1} y \in \mathcal{H},
$$

i.e., the third term also vanishes. Altogether this shows that $g \in I_{1}$ for all $g \in J$, and hence $J \subset I_{1}$. 
One might notice that Proposition 1 in [16] is a Corollary of this theorem.

Now we are ready to prove a generalization of a theorem in [6] for a compact complex homogeneous space with an invariant volume form (see also 11], [12]).

On a rational homogeneous manifold $Q$, there is a Kähler-Einstein metric, unique up to a scalar multiplier, which is invariant under a given maximal compact subgroup $K \subset$ Aut $Q$ (see [27]).

Theorem 2. Let $M$ be a connected complex compact manifold and let $G$ be a connected real Lie group acting transitively and holomorphically on $M$. Assume that $M=G / H$ admits a $G$-invariant volume element.

Then the Lie groups $I, I_{1}, J$ and $J_{1}$ are all the same and the fibers of both the global HOT-fibration and HK-fibration are connected.

Moreover, the Ricci form comes down from $G / H$ to $G / I$ to be an invariant Kähler-Einstein metric which is in the Ricci class of $G / J$.

Proof (Cf. [6]). From Proposition 5, we know that $J$ is connected. Hence, Theorem 1 implies $H \subset J_{1}=J \subset I^{0} \subset I \subset I_{1}$, where $I^{0}$ is the identity component of $I$. From Proposition 3, we know that $\mathcal{H}$ is an ideal of $\mathcal{J}$, and [34, Theorem 1] implies that $\mathcal{H}$ is an ideal of $\mathcal{I}$. Hence, $J / H^{0}$ is a complex Lie subgroup of $I^{0} / H^{0}$, where $H^{0}$ denotes the identity component of $H$. Thus, $I^{0} / J \subset G / J$ is a closed complex submanifold, and therefore a projective manifold. Since $G / J$ embeds equivariantly into $\mathbf{P}^{n}$, the maximal solvable subgroups of $\left(I^{0}\right)^{\mathbf{C}}$ have fixed points in $I^{0} / J$ by Borel's Fixed Point Theorem [21, Chapter I]. Therefore, the stablizer of $\left(I^{0}\right)^{\mathbf{C}}$ at $e J$ is parabolic, and [21, Chapter I, Theorem 6] implies that $I^{0} / J$ is a rational homogeneous space. Now we consider the two complex fibrations $I^{0} / H \rightarrow I^{0} / J$ and $I^{0} / H \rightarrow I^{0} / I^{0}$. Both fibrations have rational homogeneous spaces as bases and parallelizable homogeneous spaces as fibers. Therefore, by the uniqueness of the Tits' fibration in Propositions 4 and 5, we have $J=I^{0}$. From Part (2) of Proposition 6 , we see that $I / H$ is connected. Since $H \subset I^{0}$, this implies $J=I^{0}=I$. Now by the definition of the global HK-fibration, the Ricci form comes down to be a closed two-form on $G / I_{1}$ and the image $I_{2}$ of $I_{1}$ in $\operatorname{Aut}(G / J)$ must be a closed and open subgroup of the centralizer of an element in the Lie algebra $\mathcal{S}$ of the image of $G$ in $\operatorname{Aut}(G / J)$ (see [4, 29]). Therefore, if we can prove that $\mathcal{S}$ is compact, then by [7, Proposition 4.2], $I_{2}$ must be connected. That is, $G / J=G / I_{1}$ and hence $I_{1}=J$.

Now we consider the Ricci form on $G / J$. We have

$$
\begin{aligned}
& \operatorname{tr}_{\mathcal{G} / \mathcal{H}}(\operatorname{ad} j[x, y]-j \circ \operatorname{ad}[x, y]) \\
& \quad=\operatorname{tr}_{\mathcal{G} / \mathcal{J}}(\operatorname{ad} j[x, y]-j \circ \operatorname{ad}[x, y]) \\
& \quad+\operatorname{tr}_{\mathcal{J} / \mathcal{H}}(\operatorname{ad} j[x, y]-j \circ \operatorname{ad}[x, y]) .
\end{aligned}
$$

The second term is always zero by the definition of $J$. Therefore, the Ricci form of $G / J$ is exactly the pushdown of the Ricci form of $G / H$. But the Ricci form of $G / J$ is exactly the Ricci form of a standard Kähler-Einstein metric. By a standard result in Riemannian geometry, we have that $\mathcal{S}$ is compact. The theorem is proved.

\section{The Splitting of the Lie Algebra}

Lemma 1. Let $M$ be a compact complex homogeneous space. Let $G$ be a connected complex group of holomorphic automorphisms acting on $M$ transitively and effectively, $H$ be the isotropy subgroup, and $J=N_{G}\left(H^{0}\right)$ be the normalizer of $H^{0}$ in 
$G$. Let $G=S R$ be a Levi decomposition of $G$. Then, with respect to a Cartan subalgebra in $\mathcal{S} \cap \mathcal{J}, \mathcal{H}$ decomposes into eigenspaces.

If $h \in \mathcal{H}$ is an eigenvector with a nonzero eigenvalue, then $h=h_{s}+h_{r}$ such that $h_{s} \in \mathcal{S} \cap \mathcal{H}$ and $h_{r} \in \mathcal{R} \cap \mathcal{H}$.

Proof. Since $J \cap S$ is parabolic, its Lie algebra contains a Cartan subalgebra in $\mathcal{S}$. Since $\mathcal{H}$ is an ideal of $\mathcal{J}$, it must be decomposed into its eigenvector spaces.

If $h$ is an eigenvector with nonzero eigenvalue such that $h$ is not in $\mathcal{R}$, then there is an $s=\operatorname{sl}(2, \mathbf{C})$ generated by root vectors in $\mathcal{S}$ such that $h=h_{s}+h_{r}$ and $h_{s} \in s, h_{r} \in \mathcal{R}$ with weight $\alpha$.

If $h_{r} \neq 0$, then there is an $h_{r}^{-} \in \mathcal{R}$ which is an eigenvector with weight $-\alpha$ such that $\left[h_{s},\left[h_{s}, h_{r}^{-}\right]\right]=-h_{r}$. We have $h_{r}, h_{r}^{-} \in \operatorname{nil}(\mathcal{G})$ and

$$
\begin{aligned}
h+ & {\left[h,\left[h, h_{r}^{-}\right]\right]=h_{s}+h_{r}+\left[h_{s},\left[h_{s}, h_{r}^{-}\right]\right] } \\
& +\left[h_{r},\left[h_{s}, h_{r}^{-}\right]\right]+\left[h_{s},\left[h_{r}, h_{r}^{-}\right]\right]+\left[h_{r},\left[h_{r}, h_{r}^{-}\right]\right] \\
& =h_{s}+\left[h_{r}, h_{1}\right]+\left[h_{r}^{-}, h_{2}\right] \\
& =h_{s}+h_{r}^{2} \in \mathcal{H}
\end{aligned}
$$

where $h_{1}, h_{2} \in \operatorname{nil}(\mathcal{G}):=n$. Hence $h_{r}^{2} \in[n, n]:=n_{2}$. In this way, we can find $h_{r}^{k} \in n_{k}:=\left[n_{k-1}, n_{k-1}\right]$ such that $h_{s}+h_{r}^{k} \in \mathcal{H}$. By $n$ being nilpotent, we obtain that $h_{s} \in \mathcal{H}$, and hence $h_{r} \in \mathcal{H}$ also.

Lemma 2. Let $M=G / H$ be a compact complex homogeneous space and $G^{\mathbf{C}}$ be the minimal conplex Lie group in $\operatorname{Aut}(M)$ that contains $G$, and $G^{\mathbf{C}}=S^{\mathbf{C}} R^{\mathbf{C}}$ be a complex Levi decomposition. If the image of $G$ in $\operatorname{Aut}(G / J)$ is compact, then all the root vectors in the nil radical of $p=\mathcal{S}^{\mathbf{C}} \cap \mathcal{J}^{\mathbf{C}}$ are in $\mathcal{H}^{\mathbf{C}}$.

Proof. Let $s_{1}$ be the semisimple Lie algebra that contains all the simple factors of $s=\mathcal{G} \cap \mathcal{S}^{\mathbf{C}}$ acting nontrivially on $G / J$ and $s=s_{1}+s_{2}$. We know that $\mathcal{S}^{\mathbf{C}} \cap \mathcal{J}$ is a centralizer of an element $w$ of $s_{1}$ in $s$ as we can see in the proof of Theorem 2. Choose a Cartan subalgebra in $\mathcal{S} \cap \mathcal{J}$ and an order in its complexification such that $\mathcal{S}^{\mathbf{C}} \cap \mathcal{J}^{\mathbf{C}}$ contains a Borel subalgebra. Let $e_{\alpha}$ be a positive root vector in $s_{1}$ such that $(w . \alpha) \neq 0, X=e_{\alpha}+e_{-\alpha}$. Then on $G / J, j X=i\left(e_{\alpha}-e_{-\alpha}\right)$ and $X+i j X=2 e_{-\alpha}(\bmod i(\mathcal{J} \cap \mathcal{S}))$ is an element in $p$. But we also have $x+i j x \in \mathcal{H}^{\mathbf{C}}$, where $x$ is the corresponding element of $X$ in $\mathcal{G}$ (see the end of 5 in section 2). Therefore, $2 e_{-\alpha}+y=x+i j x \in \mathcal{H}^{\mathbf{C}}$ for some $y \in i(\mathcal{J} \cap \mathcal{S})+\mathcal{R}^{\mathbf{C}}$ and by Lemma 1 , we have $e_{-\alpha} \in \mathcal{H}^{\mathbf{C}}$ as desired since $e_{-\alpha}$ is not in $(\mathcal{J} \cap \mathcal{S})^{\mathbf{C}}$.

Lemma 3 (Cf. [2]). Let $M$ be as in Lemma 1 and $\mathcal{S}=s_{1}+s_{2}$ such that $s_{1}$ contains all the simple factors acting nontrivially on $G / J$. Then $\mathcal{G}=W_{1}+\cdots+W_{l}+W_{0}$ where $W_{i}$ are nontrivial $s_{1}$ irreducible representations for $1 \leq i \leq l$ and $W_{0}$ is a vector space containing all the $s_{1}$ fixed vectors. If $w_{1}, \cdots, w_{l}$ are the highest weight vectors, then they are linearly independent modulo $\mathcal{H}$. Moreover, $\operatorname{dim} W_{0} \leq$ $\operatorname{dim} J / H$.

Proof. The direct sum comes from the representation theory of semisimple Lie groups. If $w=\sum a_{i} w_{i} \in \mathcal{H}$ and $p=\mathcal{J} \cap s_{1}$, then $[p, w] \subset \mathcal{H}$ and $\left[s_{2}+\mathcal{R}, w\right] \subset \mathcal{H}$ since $\mathcal{H}$ is an ideal of $\mathcal{J}$. But $\left[B^{\prime}, w\right]=0$, where $B$ is the Borel subalgebra that is the minimal parabolic subalgebra containing all the positive root vectors. We obtain that $\left[s_{1}, w\right] \subset \mathcal{H}$. Therefore, $m_{1}=[\mathcal{G}, w] \subset \mathcal{H}$, and $\left[B, m_{1}\right]=[[B, \mathcal{G}], w] \subset m_{1}$. If we let $m_{k}=\left[\mathcal{G}, m_{k-1}\right]$ and assume that $m_{k} \subset \mathcal{H},\left[B, m_{k}\right] \subset m_{k}$, then $m_{k+1}=$ $\left[B+\mathcal{J}, m_{k}\right] \subset\left[[B, \mathcal{G}], m_{k-1}\right]+\left[\mathcal{G}, m_{k-1}\right]+\mathcal{H} \subset m_{k}+\mathcal{H} \subset \mathcal{H}$. Therefore, $w$ 
generates a $\mathcal{G}$-ideal in $\mathcal{H}$. This implies that $w=0$. Hence, all the weight vectors $w_{i}$ are linearly independent modulo $\mathcal{H}$.

All the vectors in $W_{0}$ correspond to the actions, regarded as the action on the fiber of the bundle $G / H \rightarrow G / J$, being without any fixed point and invariant under $S_{1}$, which is the subgroup of $G$ corresponding to $s_{1}$. Since $S_{1}$ acts on $G / J$ transitively, each of these vector fields is determined by its value on any fixed fiber of $G / H \rightarrow G / J$. We obtain that $\operatorname{dim} W_{0} \leq \operatorname{dim} J / H$.

Now we are ready to prove the Splitting Theorem for the Lie algebra:

Theorem 3. In the case of Lemma 2, we can apply Lemma 3 to $G^{\mathbf{C}} / H^{\mathbf{C}}$. All $W_{i}$ in Lemma 3 must be one of the simple factors in $s_{1}^{\mathbf{C}}$, i.e., $\mathcal{G}=s_{1} \oplus\left(s_{2}+\mathcal{R}\right)$ where $s_{2}$ is a complex semisimple Lie algebra and $s_{1}$ is a compact semisimple Lie algebra. Moreover, if we let $c$ be the centralizer of $w$ in $s_{1}, c_{1}$ be its center and $c_{2}$ be the center of $s_{2}+\mathcal{R}$, then $\mathcal{H}$ is a direct sum of the semisimple part of $s_{1} \cap \mathcal{J}$ and a subalgebra of $c_{1}+c_{2}$.

Proof. By Proposition 9 and Lemmas 1,2,3, we see that $W_{i}$ can only be the simple factors of $s_{1}$; otherwise, $J / H^{0}$ cannot be unimodular by considering the effects of the actions of the fundamental weights $H_{i}$.

The semisimple part of $s_{1} \cap \mathcal{J}$ is compact and hence must be in $\mathcal{H}$. Let $x \in \mathcal{H}$ be an eigenvector with weight zero. Then $x=h_{s}+h_{r}$ with $h_{s} \in c_{1}$ and $h_{r} \in\left(s_{2}+\mathcal{R}\right)$. $h_{s}$ cannot be zero; otherwise, $h_{r}$ generates an ideal of $\mathcal{G}$ in $\mathcal{H}$. Now $h_{r}$ must be in $c_{2}$; otherwise, $\left[\mathcal{J}, h_{r}\right]=[\mathcal{J}, x]$ will generate an ideal of $\mathcal{G}$ in $\mathcal{H}$. Therefore, we have the theorem.

\section{Global Structure Theorem}

Now we are able to obtain a global structure for our manifolds. First, we mention two lemmas, which I believe have been known for a long time:

Lemma 4 (Cf. [31, Theorem 6.15]). If $G$ is a complex Lie group and $H$ is a cocompact discrete subgroup of $G$, then $H$ is finitely generated.

This lemma also comes from the existence of finite triangularization for compact complex manifolds (see also 26]). We initially proved the following lemma by the methods in [32] and [12] in a situation in which we did not find a better reference, but then we found it in [36].

Lemma 5 (Cf. [32], see also [36, Cor. 3.4.14]). Let $G$ be a complex Lie group and $G / H$ be a compact complex parallelizable manifold with $H$ being discrete. If $C$ is the center of $G$, then $C /(C \cap H)$ is compact.

Theorem 4. Suppose $M=G / H$ is a compact complex homogeneous space with an invariant volume. Then $G=S_{1} G_{1}$ is a local direct product with $S_{1}$ being compact semisimple, which acts on $G / J$ transitively, and $G_{1}$ having complex semisimple part. $H=H_{s} C_{H}$ is a local direct product with $H_{s}$ being the semisimple part of $S_{1} \cap J$, which is compact, and the identity component of $C_{H}$ is in the center of $J . M$ is a holomorphic principal torus bundle over a product of a rational homogeneous space and a compact complex parallelizable manifold such that the torus action comes from the center of $J$. Conversely, any $G / H$ of this kind has an invariant volume. 
Proof. By Theorem 3, we have that $J=H_{s} C_{1} G_{1}$ where $C_{1}$ is a Lie group corresponding to $c_{1}$. Since $S_{1} \cap J=H_{s} C_{1}$ is connected, we have that $C_{1}$ is connected. We obtain that $C_{1}$ is in the center of $J$. We also have that $H_{s} \subset H$. $J / H=C_{1} G_{1} / H \cap C_{1} G_{1}$ is a compact complex parallelizable manifold. The identity component of $H \cap C_{1} G_{1}$ is in $C_{1} C_{2}$, where $C_{2}$ is the center of $G_{1} . C_{1} C_{2}$ is the center of $C_{1} G_{1}$. Now by Lemma 5 , we obtain that $J / H$ is a torus bundle over $\left(J / H^{0}\right) /\left(H / H^{0}\right)$. The torus action comes from the center $C^{1}$ of $J^{1}=J / H^{0}$. Let $N^{2}$ be the intersection of the pullback of $C^{1}$ in $J$ with $G_{1}$ and $N^{1}=C_{1} N^{2}$. Then $N^{1}$ is a nilpotent Lie group of at most two steps. Then $G / H N^{1}$ is a product of $S_{1} / H_{s} C_{1}$ and $G_{1} / N^{2}\left(H C_{1} \cap G_{1}\right)$.

Now we want to prove that $\mathcal{N}^{2}=c_{2}$. For any $n \in \mathcal{N}^{2}$, we have $[n, x] \in \mathcal{H}$ for all $x \in \mathcal{G}_{1}$ since $n$ represent a center element in $\mathcal{J}^{1}$. Therefore, $[n, x]$ generates an ideal of $\mathcal{G}$ in $\mathcal{H}$ and must be zero. We obtain $N^{2}=C_{2}$ and $N^{1}=C_{1} C_{2}$ is the center of $J$.

We can also get $M$ back, by forming the product of $M_{1}=S_{1} C_{2} / H \cap S_{1} C_{2}$ and $M_{2}=J / H$ and then taking the anti-diagonal equivalent relation $(x, y) \sim\left(x g, g^{-1} y\right)$ for all $g \in N^{1}=C_{1} C_{2}$.

Conversely, if $G / H$ is a complex homogeneous torus bundle over a product of a compact rational homogeneous manifold $Q$ and a parallelizable manifold $P$ such that $G$ is a local direct product $S_{1} G_{1}$ and acts on $Q$ as the compact Lie group $S_{1}$ as in the first part of Theorem 4, then $G$ acts on $M_{1}$ and $M_{2}$ with invariant volumes. $M$, being the quotient space of $M_{1} \times M_{2}$ by the anti-diagonal torus action, has invariant volumes.

This is the first part of our Main Theorem 1. Applying this theorem to the case in which $G$ is reductive, we get Main Theorem 2.

We also notice that the main point from which this theorem is true comes from Lemma 2, i.e., if either $S_{1}$ is compact or the root vectors in the nil radical of $\mathcal{S}_{1}^{\mathbf{C}} \cap \mathcal{J}^{\mathbf{C}}$ are in $\mathcal{H}$, then all the arguments go through. We shall therefore have the same structure theorem. Hence, we have the following theorem and the second part of our Main Theorem 1:

Theorem 5. Every compact complex homogeneous space $G / H$ with $G$ acting on $G / J$ as a compact Lie group admits an invariant volume.

We can also notice that all the compact complex homogeneous spaces with a 2-cohomology class whose top power is nonzero in the top cohomology group admit invariant volumes.

In our Theorem 4, if $G_{1}$ is a compact torus, we have the manifolds in [35]; if $S_{1}$ is trivial, we have the manifolds in 34 . Therefore, we also notice that $M_{1}$ in the proof of Theorem 4 is exactly the manifold studied in [35] (by extending the center of the Lie group as big as possible, we can always assume $C_{2}$ to be the complex torus and hence $G$ can be compact), and $M_{2}$ is exactly the manifold studied in 34. Our manifold is the quotient space of $M_{1} \times M_{2}$ by the anti-diagonal torus action. Conversely, whenever we have an $M_{1}$ in [35] and an $M_{2}$ in [34] such that there is a complex torus action on both $M_{1}$ and $M_{2}$ that comes from a complex subgroup in the center of $M_{2}$, we can construct our manifold as the quotient space of $M_{1} \times M_{2}$ by the anti-diagonal action of this given complex torus.

We shall try to classify those compact complex homogeneous spaces that do not admit any invariant volume in [14]. 


\section{Acknowledgement}

Here I take this opportunity to thank Professor S. Kobayashi for his interest in this work and encouragement, and his help in writing a latex file when I was modifying a certain part of [6]. I also thank Professor J. A. Wolf for his encouragement for this work and his help in papers 11], 12. I thank Professor A. Borel for discussions and for showing me the paper [32]. I also thank Professor A. T. Huckleberry for his effort in [19] and Professor J. Dorfmeister for leading me to this direction and showing me 20 and 24. I thank Professors F. A. Bogomolov, M. Gromov, Y. Siu, and K. Ding for their constant encouragement and support. Finally, I wish to express my thanks to the Department of Mathematics, Princeton University and to Professor W. C. Hsiang as well as NSF for their support, which made this work possible.

I also thank the referee of [15] in ERA-AMS for telling me about [17]. I also thank the referees for their helpful comments on this paper.

\section{REFERENCES}

1. J. F. Adams: Lectures on Lie Groups. W. A. Benjamin, New York, 1969. MR 40:5780

2. D. N. Akhiezer: A Bound for the Dimension of the Automorphism Group of a Compact Complex Homogeneous Space. Soobshch. Akad. Nauk. Grus. SSR (Russian) 110 (1983), 469472 .

3. D. N. Akhiezer: Homogeneous Complex Manifolds, in Several Complex Variables IV, Encyclopaedia of Math. Sci., vol. 10, (1994), 195-244.

4. A. Borel: Kählerian Coset Spaces of Semisimple Lie Groups, Proc. Nat. Acad. Sci. USA, 40 (1954) 1147-1151. MR 17:1108e

5. A. Borel and R. Remmert: Über kompakte homogene Kählersche Mannigfaltigkeiten, Math. Ann. 145 (1962), 429-439. MR 26:3088

6. J. Dorfmeister and Z. Guan: Classification of Compact Homogeneous Pseudo-Kähler Manifolds, Comment. Math. Helv. 67 (1992), 499-513. MR 93i:32042

7. J. Dorfmeister and Z. Guan: Fine Structure of Reductive Pseudo-Kählerian Spaces, Geom. Dedicata 39 (1991) 321-338. MR 92h:53081

8. J. Dorfmeister and Z. Guan: Pseudo-Kählerian Homogeneous Spaces Admitting a Reductive Transformation Group of Automorphisms, Math. Z. 209 (1992) 89-100. MR 92k:32058

9. J. Dorfmeister and K. Nakajima: The Fundamental Conjecture for Homogeneous Kähler Manifolds, Acta. Math. 161 (1988) 23-70. MR 89i:32066

10. H. Grauert and R. Remmert: Über kompakte homogene komplexe Mannigfaltigkeiten, Arch. Math. 13 (1962) 498-507. MR 26:3089

11. D. Guan: Examples of Compact Holomorphic Symplectic Manifolds Which Admit no Kähler Structure. In Geometry and Analysis on Complex Manifolds-Festschrift for Professor S. Kobayashi's 60th Birthday, World Scientific, River Edge, NJ, 1994, 63-74. MR 98h:53109

12. D. Guan: A Splitting Theorem for Compact Complex Homogeneous Spaces with a Symplectic Structure, Geom. Dedicata 63 (1996), 217-225. MR 98a:53105

13. D. Guan: Classification of Compact Homogeneous Spaces with Invariant Symplectic Structures, Electron. Res. Announc. Amer. Math. Soc. 3 (1997), 52-54. MR 99a:53065

14. D. Guan: Toward a Classification of Complex Homogeneous Spaces, preprint, 1998.

15. D. Guan: Classification of Compact Complex Homogeneous Spaces with Invariant Volumes, Electron. Res. Announc. Amer. Math. Soc. 3 (1997) 90-92. MR 98i:32056

16. J. Hano: Equivariant Projective Immersion of a Complex Coset Space with Non-degenerate Canonical Hermitian Form, Scripta Math. 29 (1973), 125-139. MR 51:3557

17. J. Hano: On Compact Complex Coset Spaces of Reductive Lie Groups, Proc. Amer. Math. Soc. 15 (1964), 159-163. MR 28:1258

18. G. Hochschild: The Structure of Lie Groups, Holden-Day, 1965. MR 34:7696

19. A. T. Huckleberry: Homogeneous Pseudo-Kählerian Manifolds: A Hamiltonian Viewpoint, Note di Matematica 10 (1990) suppl. 2, 337-342. MR 94f:53052 
20. J. Hano and S. Kobayashi: A Fibering of a Class of Homogeneous Complex Manifolds, Trans. Amer. Math. Soc. 94 (1960), 233-243. MR 22:5990

21. A. T. Huckleberry and E. Oeljeklaus: Classification Theorems for Almost Homogeneous Spaces, Publ. de l'Institut Élie Cartan, vol. 9, Nancy, Janvier 1984, 178 pages. MR 86g:32050

22. J. E. Humphreys: Introduction to Lie Algebras and Representation Theory, Graduate Texts in Mathematics, Vol. 9, Springer-Verlag, 1972. MR 48:2197

23. T. Iwamoto: Density Properties of Complex Lie Groups, Osaka J. Math. 23 (1986) 859-865. MR 88f:22023

24. T. Iwamoto: Algebraic Groups and Co-compact Subgroups of Complex Linear Groups, Memoirs of the Faculty of Sciences, Kyushu University, Ser. A, 42 (1988) 1-7. MR 89d:22005

25. S. Kobayashi: Differential Geometry of Complex Vector Bundles, Iwanami Shoten, Tokyo and Princeton University Press, 1987. MR 89e:53100

26. R. C. Kirby and L. C. Siebenmann: On the Triangulation of Manifolds and the Hauptvermutung, Bull. Amer. Math. Soc., vol. 75 (1969), 742-749. MR 39:3500

27. J. L. Koszul: Sur la Forme Hermitienne Canonique des Espaces Homogènes Complexes, Canad. J. Math. 7 (1955), 562-576. MR 17:1109a

28. A. I. Malcev: On a class of Homogeneous Spaces. Amer. Math. Soc. Transl., no. 39 (1951). MR 12:589e

29. Y. Matsushima: Sur les Espaces Homogènes Kählériens d'un Groupe de Lie Réductif, Nagoya Math. J. 11 (1957), 53-60. MR 19:315c

30. Y. Matsushima: Sur Certaines Variétés Homogènes Complexes, Nagoya Math. J. 18 (1961) 1-12. MR 25:2147

31. M. S. Raghunathan: Discrete Subgroups of Lie Groups, Ergebnisse der Math. und ihrer Grenzgebiete, Band 68 (1972). MR 58:22394a

32. A. Selberg: On Discontinuous Groups in Higher-Dimensional Symmetric Spaces, In Contributions to Function Theory, Tata Institute of Fundamental Research, Bombay, 1960, 147-164. MR 24:A188

33. J. Tits: Espaces Homogènes Complexes Compacts, Comment. Math. Helv. 37 (1962), 111-120. MR 27:4248

34. Hsien-Chung Wang: Complex Parallisable Manifolds, Proc. Amer. Math. Soc. 5 (1954), 771776. MR 17:531a

35. Hsien-Chung Wang: Closed Manifolds with Homogeneous Complex Structure, Amer. J. Math. 76 (1954) 1-32. MR 16:518a

36. J. Winkelmann: Complex Analytic Geometry of Complex Parallelizable Manifolds. Mém. Soc. Math. Fr. (N. S.) vol. 72-73 (1998). MR 99g:32058

Department of Mathematics, The University of California at Riverside, Riverside, CALIFORNiA 92521

E-mail address: zguan@math.ucr.edu 